“ (C) 2018 IEEE. Personal use of this material is permitted. Permission from IEEE must be obtained for all other uses, in any current or future media, including

reprinting/republishing this material for advertising or promotional purposes, creating new collective works, for resale or redistribution to servers or lists, or reuse of any copyrighted component of this work in other works." 


\title{
A Deformable Spiral Based Algorithm to Smooth Coverage Path Planning for Marine Growth Removal
}

\author{
Mahdi Hassan and Dikai Liu
}

\begin{abstract}
Marine growths that flourish on the surfaces of underwater structures, such as bridge pylons, make the inspection and maintenance of these structures challenging. A robotic solution, using an Intervention Autonomous Underwater Vehicle (I-AUV), is developed for removing marine growth. This paper presents a Deformable Spiral Coverage Path Planning (DSCPP) algorithm for marine growth removal. DSCPP generates smooth paths to prevent damage to the surfaces of the structures and to avoid frequent or aggressive decelerations and accelerations due to sharp turns. DSCPP generates a spiral path within a circle and analytically maps the path to a minimum bounding rectangle which encompasses an area of a surface with marine growth. It aims to achieve a spiral path with minimal length while preventing missed areas of coverage. Several case studies are presented to validate the algorithm. Comparison results show that DSCPP outperforms the popular boustrophedonbased coverage approach when considering the requirements for the application under consideration.
\end{abstract}

\section{INTRODUCTION}

Removing marine growth from underwater structures is a tedious and expensive task, and it is a problem on a global scale. Examples of marine growth include barnacles, algae, and seaweed. Marine growth removal task is mostly carried out manually by a human diver using a high-pressure water gun. This manual operation raises two main concerns: 1) divers are exposed to significant health and safety risks, and 2) human fatigue and poor performance are likely when operating for extended periods of time. Thus, using an IAUV is an attractive alternative option (Fig. 1).

This paper addresses the coverage path planning problem [1] for an I-AUV to remove marine growth from surfaces of underwater structures. That is, coverage paths are generated such that the marine growth is removed by directing the highpressure water stream along the paths. The water stream is directed along the path using a cleaning tool (e.g. a nozzle) mounted to the end-effector of the cleaning manipulator (Fig. 1).

There are unique requirements and conditions associated with the problem under consideration. One main requirement is to prevent the water stream from damaging the surface. Thus, properties such as the blasting stream pressure from the water jet and blasting spot traversal speed along the path should be determined [2] such that they are appropriate for the removal of the marine growth and yet not cause damage to the surface. In this paper, it is assumed that these parameters are given. The local exposure time of the surface to the water jet should not be sufficient to cause fatigue

Authors are from the Centre for Autonomous Systems (CAS) at the University of Technology Sydney, 15 Broadway, Ultimo NSW 2007, Australia Mahdi.Hassan@uts. edu . au

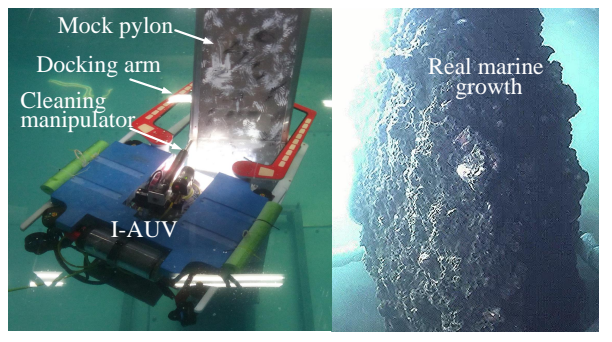

Fig. 1: The SPIR robot, an I-AUV for marine growth removal, developed in the Centre for Autonomous Systems (CAS) at the University of Technology Sydney (UTS).

cracks in the structure [3]. Thus, the traversal speed is set high enough to prevent fatigue cracks and allow multiple passes if needed without damaging the surface. The aim is to generate a smooth coverage path to keep the blasting spot traversal speed at the given value. Smooth paths prevent the aggressive slowing down of the blasting spot at sharp turns and causing prolonged local exposure of the surface to the water jet.

The DSCPP algorithm deforms a spiral path to fit within any size rectangle. It appropriately maps a smooth spiral path generated inside a circle to a spiral path inside a Minimum Bounding Rectangle (MBR). Given an area representing a surface of marine growth, the MBR is the smallest rectangle that fits the area. The mapping of the spiral is carried out analytically between a circle and a square using Fernandez-Guasti squircular mapping (FG-mapping) [4]. However, modifications are made using control points to facilitate mapping between a circle and any size rectangle instead of a square. The entire MBR doesn't need to be covered, i.e. the spiral path within an MBR can end once the area representing the surface of the marine growth is covered. The creation of the spiral path and the mapping to an MBR are done such that: 1) the spiral path remains smooth while the path length is minimized, and 2) missed areas are prevented by maintaining an acceptable gap between any two adjacent laps of the spiral. There may be partial overlaps between any two successive laps of the spiral path; however, this overlap is a trade-off to having a smooth path. As explained previously, having multiple passes with high enough blasting spot traversal speed is better than a single pass with a traversal speed that is below the threshold for preventing fatigue cracks.

There are a limited number of research works that incorporate spiral-like paths [5]-[12]. In [6], spiral paths, wall-following, and virtual wall path tracking methods are 
combined to produce the final path. On-line methods based on linked spiral paths are presented in [7] and [8] where wallfollowing and iterative linking of spiral paths is considered. A backtracking spiral algorithm is presented in [11] where spiral paths are generated for simple regions and then linked together using a backtracking strategy. An extension of this algorithm was proposed in [12] where wall-following and return path by a virtual pipe were introduced to the original algorithm. Although the above algorithms aim to obtain smooth paths, they don't completely prevent sharp turns. That is, due to procedures such as wall-following and virtual wall path tracking, the resulting path may still have many sharp turns. This is acceptable for some applications, particularly for on-line applications, since there are obstacles in the environment and the path needs to be strictly within the boundary of the target area. However, for the application under consideration, the coverage path can have some degree of flexibility in terms of drifting outside the boundary of the area and a smooth path is required.

The DSCPP algorithm takes advantage of approximating the surface of marine growth using an MBR to provide a smoother path. The novelty of the DSCPP algorithm is that it deforms a spiral path to fits within any size rectangle, and a mapping strategy is used to map a spiral path within a circle to a path within a rectangle such that the spiral gradually morphs to the boundary and satisfies the relevant constraints and requirements. The resulting path is smooth, i.e. at least $G^{1}$ continuous since the tangent direction is the same for the joint point of any two segments of the path.

\section{PROBLEM DEFINITION}

Let $X=\left\{\boldsymbol{x}_{1}, \boldsymbol{x}_{2}, \ldots, \boldsymbol{x}_{M}\right\}$ be a given set of points that represents a surface with marine growth. It is assumed that the map of the marine growth is given; e.g., through SLAM [13]. It is also assumed that the I-AUV can navigate and localize itself. At a given I-AUV body position, the I-AUV can cover a set of points $\mathcal{X} \subseteq X$ which represent part of the marine growth surface. Let $\delta$ be the effective cutting length of the blasting stream exiting the cleaning nozzle. Thus, when the nozzle is directed along a coverage path, it can remove at least a thickness of $\delta$ from the marine growth.

The problem is to plan a coverage path to cover a set of points $\mathcal{X}$ for each given I-AUV body position. There are two main requirements. The first requirement is to generate a smooth path. As discussed in the introduction, a smooth path with no sharp corners prevents the traversal speed of the blasting spot along the path to reduce aggressively at the corners and cause damage to the surface. The second requirement is to prevent missed areas and to aim at shortening the length of the smooth path as much as possible without negatively impacting the smoothness of the path.

\section{THE DSCPP ALGORITHM}

The DSCPP algorithm generates a non-uniform arithmetic spiral as shown in Fig. 2 (left). The spiral path is mapped to fit within a given Minimum Bounding Rectangle (MBR) [14] such that the length of the spiral path is minimized and missed areas are prevented. Missed areas are caused if the gap between any two consecutive laps of the spiral path exceeds the maximum allowable gap. At a given I-AUV body position, an MBR fits the points in $\mathcal{X}$. The aim is to tailor the algorithm to generate a smooth path for any size rectangle as opposed to an arbitrary shape that perfectly fits the points in $\mathcal{X}$. The MBR is perpendicular to the heading direction of the I-AUV. The cleaning arm will follow the spiral path generated within the MBR to blast away a thickness of $\delta$ from the marine growth. Using MBRs has some advantages. If the marine growth covers a large area on the surface of the structure, then many MBRs can be stacked around each other to cover the large area without overlap or gap between the MBRs. On the other hand, if the area on the MBR that represents the marine growth surface doesn't occupy the entire MBR, then the spiral path which starts at the center of an MBR can stop once the area is covered or a threshold on coverage is met.

\section{A. The Mapping Strategy}

Let a space within a circle centered at the origin be defined as $\mathrm{O}=\left\{(u, v) \in \mathbb{R}^{2} \mid u^{2}+v^{2} \leq 1\right\}$ where $u$ and $v$ are coordinates in $\mathrm{O}$. Similarly, let a space within a square be defined as $\mathrm{S}=\left\{(x, y) \in \mathbb{R}^{2}|| x|\leq 1| y \mid, \leq 1\right\}$ where $x$ and $y$ are coordinates in $\mathrm{S}$.

Fernandez-Guasti squircular mapping (FG-mapping) [4] is considered for mapping the spiral from the O-space to the $\mathrm{S}$ space, as shown in Fig. 2. This analytical method of mapping between $\mathrm{O}$-space and $\mathrm{S}$-space is fast and convenient, and enables a smooth spiral to be generated in the S-space such that it gradually morphs to the boundary of the MBR.

Using the FG-mapping, given the $u$ and $v$ coordinates of a point, $\boldsymbol{p}$, in $\mathrm{O}$-space, the corresponding $x$ and $y$ coordinates of the point in S-space can be calculated as

$$
\begin{aligned}
& \boldsymbol{p}_{x}=\mathrm{O} 2 \mathrm{~S}(u, v)=\frac{\operatorname{sgn}(u, v)}{v \sqrt{2}} \sqrt{u^{2}+v^{2}-\sqrt{\left(u^{2}+v^{2}\right)\left(u^{2}+v^{2}-4 u^{2} v^{2}\right)}} \\
& \boldsymbol{p}_{y}=\mathrm{O} 2 \mathrm{~S}(u, v)=\frac{\operatorname{sgn}(u, v)}{u \sqrt{2}} \sqrt{u^{2}+v^{2}-\sqrt{\left(u^{2}+v^{2}\right)\left(u^{2}+v^{2}-4 u^{2} v^{2}\right)}}
\end{aligned}
$$

and conversely, for mapping from S-space to O-space:

$$
\begin{aligned}
& \boldsymbol{p}_{u}=\operatorname{S} 2 \mathrm{O}(x, y)=x \sqrt{x^{2}+y^{2}-x^{2} y^{2}} / \sqrt{x^{2}+y^{2}}, \\
& \boldsymbol{p}_{v}=\operatorname{S} 2 \mathrm{O}(x, y)=y \sqrt{x^{2}+y^{2}-x^{2} y^{2}} / \sqrt{x^{2}+y^{2}} .
\end{aligned}
$$

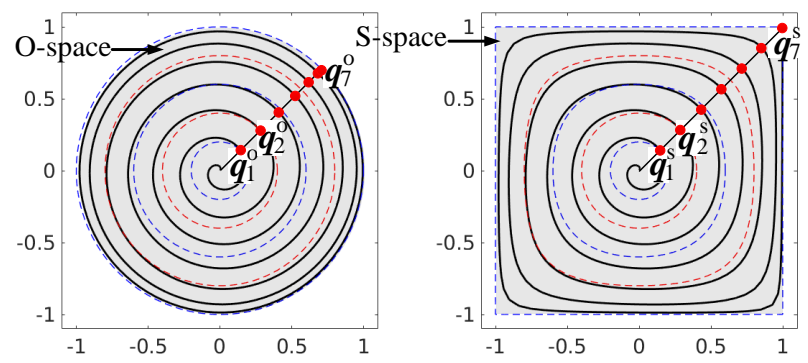

Fig. 2: Mapping a spiral from $\mathrm{O}$-space to $\mathrm{S}$-space. To visualize the mapping behavior, the equally spaced dashed circles in O-space map to the dashed squircles in S-space. 


\section{B. Generating a Spiral Path with Minimal Length}

A pseudo-code of DSCPP is presented in Alg. 11 The inputs to the algorithm are the MBR's width, $w^{\mathrm{M}}$; the MBR's height, $h^{\mathrm{M}}$; and the maximum allowable gap between adjacent laps, $g^{\max }$. The value of $g^{\max }$ is determined empirically based on the cleaning spot size of the blasting stream. The algorithm returns a sequence, $P=\left\{\boldsymbol{p}_{1}, \boldsymbol{p}_{2}, \ldots, \boldsymbol{p}_{N}\right\}$, of points along the spiral path (line 22,

The equation for the normal arithmetic spiral (also referred to as Archimedean spiral) in polar coordinates $(r, \theta)$ is

$$
r=a+b \theta
$$

where $r$ is the length of the radius from the origin, $\theta$ is the angular position, $a$ and $b$ are constants that define the initial radius and the gap between laps, respectively.

Describing the spiral in Cartesian coordinates will yield

$$
r_{x}=r \cos (\theta), \quad r_{y}=r \sin (\theta)
$$

where $r_{x}$ and $r_{y}$ are the $x$ and $y$ coordinates of $r$, respectively.

Let $r_{1}$ and $r_{2}$ be the radius from the origin at the start of the first and second lap, respectively, and $g$ be the desired gap between the two laps. Starting the spiral at the origin (i.e. when $\theta=0$ and $a=0$ ), then $r_{1}=0$. After a full rotation (when $\theta=2 \pi$ ), $r_{2}=0+2 b \pi$ and $g=r_{2}-r_{1}=r_{2}$. Thus,

$$
b=\frac{g}{2 \pi} \text {. }
$$

Substituting Eq. (5) into Eq. (3), and then substituting the

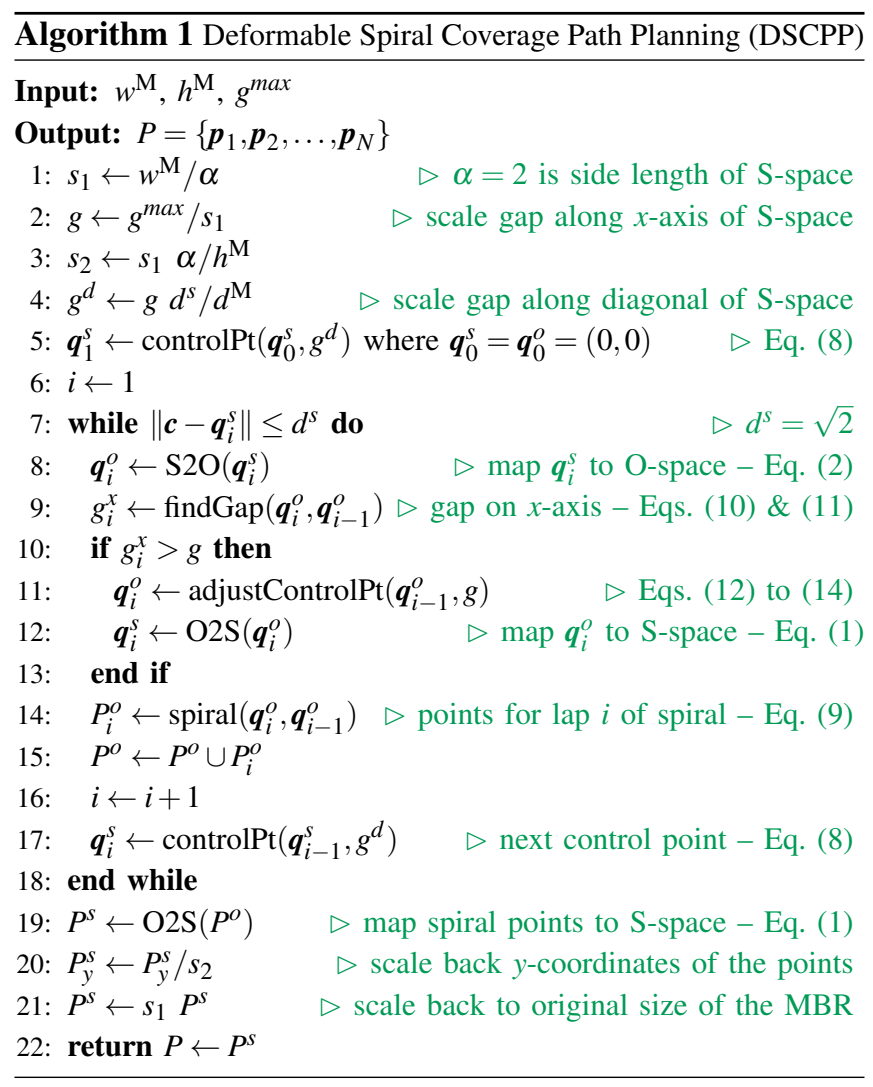

result into Eq. (4) would yield:

$$
r_{x}=\left(a+g \frac{\theta}{2 \pi}\right) \cos (\theta), \quad r_{y}=\left(a+g \frac{\theta}{2 \pi}\right) \sin (\theta) .
$$

For this subsection, suppose that an MBR occupies the entire S-space. Lines 1 to 4 as well as lines 9 to 13 of Alg. 1 are relevant to the next subsection where the MBR does not occupy the entire $\mathrm{S}$-space.

The aim is to create a spiral with a minimal number of laps so as to obtain a minimal length spiral. Let $\boldsymbol{c}$ and $\boldsymbol{e}$ be the centroid and any corner vertex of the S-space, respectively. A spiral that covers the $\mathrm{S}$-space has minimal number of laps if the gap between any two consecutive laps on the line segment $\overline{\boldsymbol{c e}}$ is maximal, i.e. $g_{i}=g^{\max } \forall i=1,2, \ldots, n$ where $g_{i}$ is the gap between the $i$ th and $(i-1)$ th laps of the spiral at the intersection with $\overline{\boldsymbol{c e}}$. Thus, the number of laps of the spiral is ideally $n=\|\boldsymbol{c}-\boldsymbol{e}\| / g^{\max }$. As an example, Fig. 2 shows a spiral path in the $\mathrm{S}$-space with a minimal number of laps.

Control points, $\boldsymbol{q}_{i}^{s}$ for $i=1,2, \ldots, n$, are introduced (lines 5 and 17 of Alg. 11 along the diagonal of the S-space, i.e. along $\overline{\overline{c e}}$, e.g. as shown in Fig. 2. The spiral path will pass through these control points to satisfy the gap requirement explained above. That is,

$$
\left\|\boldsymbol{q}_{i}^{s}-\boldsymbol{q}_{i-1}^{s}\right\|=g
$$

where $g$ is the gap between any two consecutive laps of the spiral along $\overline{\boldsymbol{c e}}$, and $g=g^{\max }$ for an MBR that occupies the entire S-space.

From Eq. (7), given $g$, then $\boldsymbol{q}_{i}^{s}$ can be calculated iteratively for all $i=1,2, \ldots, n$ :

$$
\left(\boldsymbol{q}_{i}^{s}\right)_{x}=\left(\boldsymbol{q}_{i-1}^{S}\right)_{x}+g \cos (\pi / 4), \quad\left(\boldsymbol{q}_{i}^{s}\right)_{y}=\left(\boldsymbol{q}_{i-1}^{s}\right)_{y}+g \sin (\pi / 4)
$$

where $\boldsymbol{q}_{0}^{s}=(0,0)$ and the angle $\pi / 4$ is because the control points are on the diagonal (at $45^{\circ}$ to the horizontal axis).

To generate the $i$ th lap of the spiral, the control point $\boldsymbol{q}_{i}^{s}$ is first mapped from the $\mathrm{S}$-space to O-space (line 8, i.e. $\boldsymbol{q}_{i}^{o}=\mathrm{S} 2 \mathrm{O}\left(\boldsymbol{q}_{i}^{s}\right)$ using Eq. (2). In the O-space, the $i$ th lap of the spiral path starts at $\boldsymbol{q}_{i-1}^{o}$ and ends at $\boldsymbol{q}_{i}^{o}$ (line 14). From Eq. (6), the points, $P_{i}^{o}=\left\{\boldsymbol{p}_{i, 1}^{o}, \boldsymbol{p}_{i, 2}^{o}, \ldots, \boldsymbol{p}_{i, m}^{o}\right\}$, representing the $i$ th lap of the spiral in O-space are calculated as follow:

$$
\begin{aligned}
& \left(\boldsymbol{p}_{i, j}^{o}\right)_{u}=\left(a+g_{i}^{o} \frac{\theta}{\pi / 4+2 \pi}\right) \cos (\theta), \\
& \left(\boldsymbol{p}_{i, j}^{o}\right)_{v}=\left(a+g_{i}^{o} \frac{\theta}{\pi / 4+2 \pi}\right) \sin (\theta),
\end{aligned}
$$

for $j=1,2, \ldots, m$, where $\theta=\frac{\pi}{4}+\frac{j}{m} 2 \pi ; a=\left\|\boldsymbol{c}-\boldsymbol{q}_{i-1}^{o}\right\|$ is the distance from the origin $\boldsymbol{c}$ to the previous control point $\boldsymbol{q}_{i-1}^{o}$, and $g_{i}^{o}=\left\|\boldsymbol{q}_{i}^{o}-\boldsymbol{q}_{i-1}^{o}\right\|$ is the gap between the $i$ th and $(i-1)$ th lap of the spiral in O-space. Note that $g_{i+1}^{o}<g_{i}^{o} \forall i$, resulting in a non-uniform spiral in the $\mathrm{O}$-space so as to maintain a constant gap size of $g$ along the diagonal in the S-space.

The points, $P_{i}^{o}$, representing the $i$ th lap are then added to $P^{o}$ (line 15). The above procedure is repeated if the next control point, $\boldsymbol{q}_{i+1}^{s}$, is within the S-space (line 7). Finally, the points in $P^{o}$ are mapped to S-space (line 19. The points can be joined by a smooth curve using a spline if needed. 


\section{Mapping to an $M B R$}

The FG-mapping is between O-space (circle) and S-space (square); however, the MBR can be rectangular in shape. One option to handle this issue of mapping to an MBR is to scale the MBR to fit inside the S-space while maintaining its aspect ratio, then creating the spiral in the region of the O-space that maps nicely to the scaled MBR in the S-space. However, finding this region in the O-space is not trivial and may no longer be a circle to enable creating a simple arithmetic spiral. A simpler alternative is to scale the MBR such that it occupies the entire S-space. Thus, the spiral path can be generated to occupy the entire O-space as per the procedure in the previous subsection. However, this option requires satisfying the maximum gap constraint as per below.

To fit the MBR inside the S-space, it is first scaled by a factor of $s_{1}=w^{\mathrm{M}} / \alpha$ (line 1 of Alg. 1) where $\alpha=2$ is the side length of the S-space. Note that in Alg. 1 the width $w^{\mathrm{M}}$ is always considered to be the longer side of the MBR and it is rotated to align with the $x$-axis of the S-space. Let $d^{\mathrm{M}}$ be the length of the line segment from the centroid to the corner of this scaled MBR. The MBR is then scaled up only along the height by a factor of $s_{2}=s_{1} \alpha / h^{\mathrm{M}}$ (line 3 to encompass the entire $\mathrm{S}$-space. Let $d^{s}$ be the length of the diagonal line segment from the centroid, $\boldsymbol{c}$, to any corner, $\boldsymbol{e}$, of the S-space. That is, $d^{s}=\sqrt{2}$ since $S=\left\{(x, y) \in \mathbb{R}^{2}|| x|\leq 1| y \mid, \leq 1\right\}$. The gap size is important along the diagonal to obtain a spiral with a minimal number of laps, as explained in the previous subsection. Thus, the gap size is scaled with respect to the diagonal, i.e. $g^{d}=g d^{s} / d^{\mathrm{M}}$ (line 4 where $g^{d}$ is the maximum gap along the diagonal of the scaled MBR that occupies the entire S-space, and $g=g^{\max } / s_{1}$ (line 2). After generating the spiral path in the $\mathrm{S}$-space, the path is scaled back by a factor of $s_{2}$ along the $y$-axis only (line 20 and further scaled by a factor of $s_{1}$ along both axes (line 21). The MBR is also transformed appropriately to match the original pose of the MBR.

When scaling back along the $y$-axis, the gap in the $x$ axis doesn't change which may cause the gaps to exceed the maximum allowable gap. Therefore, the gap, $g_{i}^{x}$ (line 9, along the $x$-axis needs to be checked (line 10 for each lap and if $g_{i}^{x}>g$ (line 10, then the control point $\boldsymbol{q}_{i}^{o}$ is adjusted (line 11) such that it results in $g_{i}^{x}=g$.

The gap, $g_{i}^{x}$ (line 9), along the $x$-axis is

$$
g_{i}^{x}=g_{i}^{u}=\left\|\rho_{i}-\rho_{i-1}\right\|
$$

where $\rho_{\mathbf{i}}$ and $\rho_{\mathbf{i}-\mathbf{1}}$ are the points on the spiral that intersect with the positive $x$-axis at the $i$ th and $(i-1)$ th lap, respectively. Any point on the $x$-axis of S-space maps to the same point on the $u$-axis of O-space, and vice versa; hence $g_{i}^{x}=g_{i}^{u}$.

The $v$-coordinate of the points $\rho_{\mathbf{i}}$ for $i=1,2, \ldots, m$ is zero, i.e. $\left(\rho_{i}\right)_{v}=0$, since these points are on the $u$-axis; whereas $\left(\rho_{i}\right)_{u}$ can be calculated as

$$
\left(\rho_{i}\right)_{u}=a+\frac{7}{8} g_{i}^{o}
$$

where $a=\left\|\boldsymbol{c}-\boldsymbol{q}_{i-1}^{o}\right\|$ is the initial radius of the spiral at the $i$ th lap, and $g_{i}^{o}=\left\|\boldsymbol{q}_{i}^{o}-\boldsymbol{q}_{i-1}^{o}\right\|$ is the gap on the diagonal of the O-space, and $\boldsymbol{q}_{i}^{o}=\mathrm{S} 2 \mathrm{O}\left(\boldsymbol{q}_{i}^{s}\right)$. Equation 11 means that the $i$ th lap of the spiral will intersect the $x$-axis at the point $\rho_{i}$ after $7 / 8$ of a turn (i.e. $\frac{2 \pi-(\pi / 4)}{2 \pi}=\frac{7}{8}$ ) since each lap starts and ends on the diagonal.

If $g_{i}^{x}$ in Eq. (10) is greater than the maximum gap, $g$ (line 10 of Alg. 1), then the control point, $\boldsymbol{q}_{i}^{o}$, needs to be adjusted to achieve $g_{i}^{x}=g_{i}^{u}=g$. In this case, from Eq. 10. and according to line 11 of Alg. 1 .

$$
\left(\rho_{i}\right)_{u}=\left(\rho_{i-1}\right)_{u}+g,
$$

and from Eq. (11)

$$
g_{i}^{o}=\frac{\left(\rho_{i}\right)_{u}-a}{7 / 8}
$$

and finally, similar to Eq. (8),

$\left(\boldsymbol{q}_{i}^{o}\right)_{u}=\left(\boldsymbol{q}_{i-1}^{o}\right)_{u}+g_{i}^{o} \cos (\pi / 4), \quad\left(\boldsymbol{q}_{i}^{o}\right)_{v}=\left(\boldsymbol{q}_{i-1}^{o}\right)_{v}+g_{i}^{o} \sin (\pi / 4)$

which are substituted into Eq. (9) for $i$ th lap of the spiral.

\section{Smoothness}

As shown in Fig. 2, the spiral created in the S-space gradually becomes less smooth as it morphs to the boundary of the MBR. The rate of coverage of the MBR over time is also not linear. That is, the spiral path covers less and less of the MBR as it morphs to the boundary. Thus, the path can be made smoother and significantly reduced in length by making the spiral to stop when a coverage threshold is met or when all points representing the surface of the marine growth are covered. By definition, arithmetic spirals have continuous tangent; thus, at least $G^{1}$ continuous. A spiral centered at the origin of the $\mathrm{O}$-space also have tangent continuity in the $\mathrm{S}$ space as shown in Fig. 2

\section{CASE STUDIES}

Three case studies are presented to validate DSCPP. First DSCPP is tested for a real-world application, then it is tested for MBRs with different aspect ratios, and finally, it is compared to the popular boustrophedon coverage.

\section{A. Case Study 1: marine growth removal from a bridge pylon}

The aim here is test DSCPP for the real-world application of marine growth removal from bridge pylons.

1) Background: The SPIR robot that was shown in Fig. 1 is an example I-AUV and designed for marine growth removal from bridge pylons. The SPIR robot is docked to the pylon using the two docking arms while removing the marine growth to prevent it from drifting away due to disturbances such as strong water currents. At first, a global map of the marine growth and the pylon can be obtained through SLAM [13] and prior knowledge of the pylon. The volume representing the marine growth is partitioned using $K$ Bounding Volumes (BVs). The partitioning method considered creates BVs such as those shown in Fig. 4 Let $L$ vertical partitioning planes, $l_{1}, l_{2}, \ldots, l_{L}$, be placed radially around the pylon. A bounding volume, $v_{k}(k \in\{1,2, \ldots, K\})$, is radially bounded by two neighboring planes $l_{i^{\prime}}$ and $l_{j^{\prime}}$ with corresponding angles $\psi\left(l_{i^{\prime}}\right)$ and $\psi\left(l_{j^{\prime}}\right)$; and vertically 

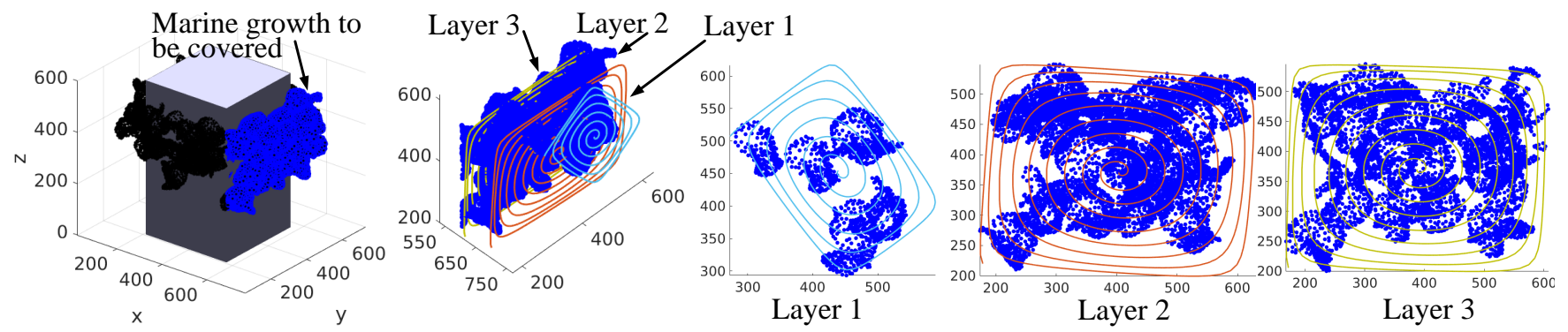

Fig. 3: Spiral paths created for three layers of a BV where the BV contains the highlighted points in the figure.

bounded by $z^{\min }\left(v_{k}\right)$ and $z^{\max }\left(v_{k}\right)$. Using this partitioning method, checking if a point is within a BV is fast and convenient particularly if cylindrical coordinates are used. For the case where a BV is not completely reachable by an I$\mathrm{AUV}$, then the BV can be iteratively divided into smaller BVs using additional vertical partitioning planes, e.g. as shown in Fig. 4 The visiting sequence of the BVs by the I-AUV can be optimized, e.g. using optimization-based approaches [15], [16].

The partitioning strategy enables simple and convenient conversion of each BV to a number of rectangular layers. For each BV, the aim is to generate layers that are parallel to the pylon's surface (or the relevant face of the pylon) as shown in Fig. 4. For cylindrical pylons or curved surfaces, the curved surface can be converted to an equivalent rectangle, $R$, by unwrapping the curved surface. An MBR can then be generated within $R$, and the spiral path within the MBR can then be mapped back to the curved layer. A new scan may be performed prior to blasting each layer for a more accurate map of the marine growth.

2) Results: A mock pylon with marine growth is used to test the DSCPP algorithm and to provide a visual demonstration of the results. Suppose a V-shaped BV contains the points highlighted in Fig. 3 . The marine growth in the BV is divided into three layers and an MBR fits the points for each layer. A spiral path is created for the MBR of each layer (shown in Fig. 3 ) as per DSCPP. The maximum gap between laps is set to $30 \mathrm{~mm}$, and the spiral paths conform to this gap requirement. Note that it may be beneficial to

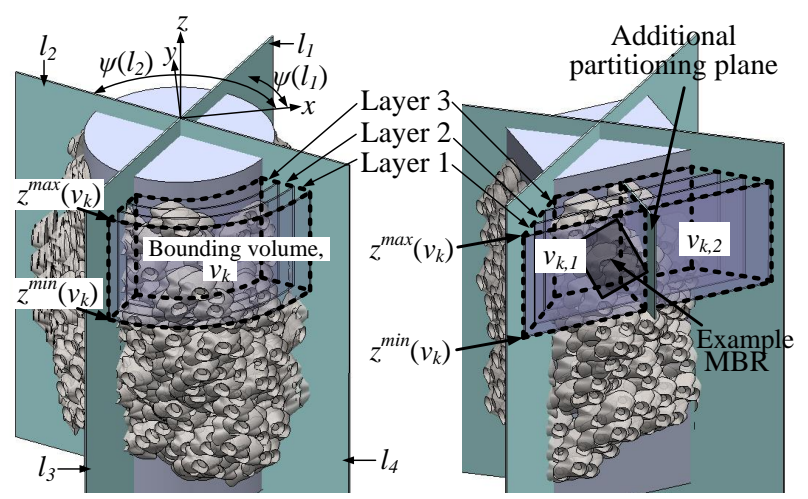

Fig. 4: Examples of partitioning the volume representing the marine growth. divide the points in each layer into a number of clusters and cover each cluster independently using an MBR. However, investigating this option for improving the performance is not the focus of the paper.

\section{B. Case Study 2: spiral on MBRs with various aspect ratios}

The DSCPP algorithm is applied to MBRs with various aspect ratios, and paths lengths are compared to ideal path lengths. An ideal spiral path (lower bound on optimum) is a spiral that has the shortest possible length with number of laps $n=\|\boldsymbol{c}-\boldsymbol{e}\| / g^{\max }$ (Section III-B) that fits within a given MBR. However, to satisfy the gap constraint, the length of the actual spiral path may be longer (Section [III-C). Figure 5 is constructed to quantify how much longer the actual spiral paths are from the ideal spiral paths for MBRs with various aspect ratios. MBRs are created by fixing the width at 2 units (width of the S-space) while ranging the height from 0 to 2 in steps of 0.002 (giving 1000 MBRs). The maximum gap, $g$, between laps is set to 0.0002 . The large number of MBRs and the very small value of $g$ is to obtain high accuracy and resolution for the graph. Figure 5 shows that the difference between the actual length and the ideal length of the spirals decreases as the MBR approaches the 2:2 aspect ratio (width:height ratio). The maximum difference is less than 9\%. Some other existing coverage paths (e.g. the boustrophedon path) may be shorter in length. However, these coverage paths may not be smooth, as discussed in the introduction. Thus, the aim here has been to compare the length with an ideal spiral path length. For very thin MBRs, the spiral path may not be very smooth and alternative smooth paths may be investigated. However, such thin MBRs are unlikely for the application under consideration.
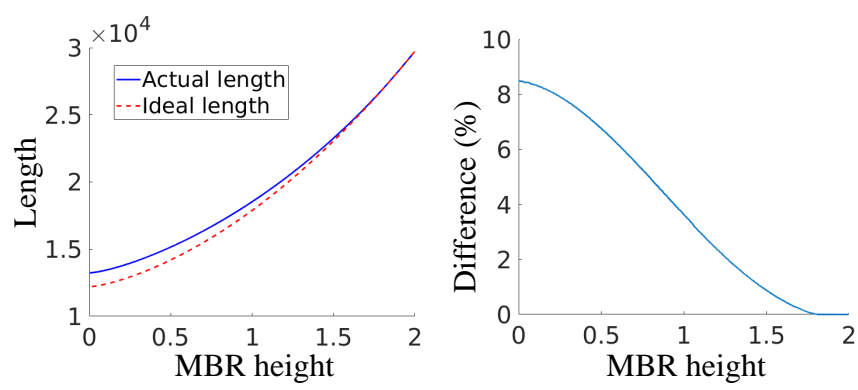

Fig. 5: Difference of actual spiral length to ideal length. 

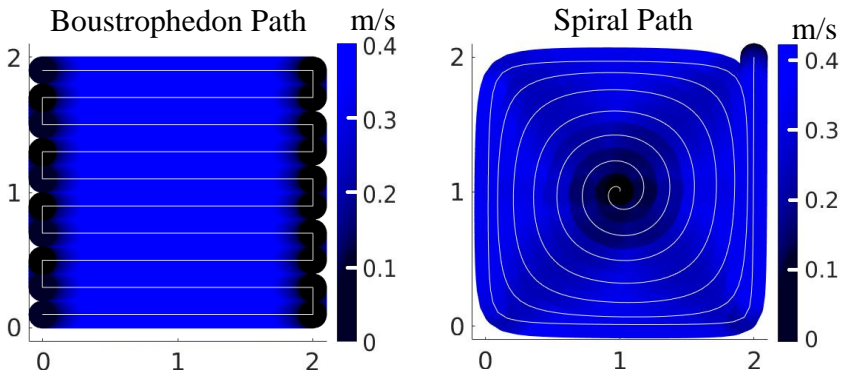

Fig. 6: Blasting spot velocity profile for the boustrophedon path and the spiral path.

\section{Case Study 3: comparison with a boustrophedon path}

The DSCPP algorithm is compared to the popular boustrophedon path planner (Fig. 6). Suppose that the desired traversal speed of the blasting spot along the path is $0.4 \mathrm{~m} / \mathrm{s}$ and that the maximum speed in both the $x$ and $y$ directions is $0.4 \mathrm{~m} / \mathrm{s}$, with a maximum acceleration of $0.4 \mathrm{~m} / \mathrm{s}^{2}$. As shown in Fig. 7. the boustrophedon path causes large acceleration and deceleration of the blasting spot, and the velocity reduces to near-zero at all corners (Fig. 6). This causes the regions around the corners to receive a significantly longer period of blasting which may damage the surface. However, the spiral path shown in Fig. 6 causes the velocity to remain largely near the desired velocity (Fig. 7). Although the spiral path takes longer to execute (Fig. 7) and causes partial overlaps between successive laps, the objective here is to prevent damage to the surface due to operation at lower speeds.

\section{CONCLUSION}

A Deformable Spiral Coverage Path Planning (DSCPP) algorithm was presented for marine growth removal from underwater structures. The DSCPP algorithm creates smooth paths to prevent damage to the structure and to avoid frequent and aggressive decelerations and accelerations due to sharp turns. The DSCPP algorithm appropriately maps a smooth spiral generated within a circle to fit within a Minimum Bounding Rectangle (MBR) using a simple, fast and analytical mapping strategy named Fernandez-Guasti squircular mapping. In doing so, the aim has been to achieve a spiral path with minimal length and to prevent missed areas of coverage. Case studies demonstrated the effectiveness of the DSCPP algorithm and showed that the difference between

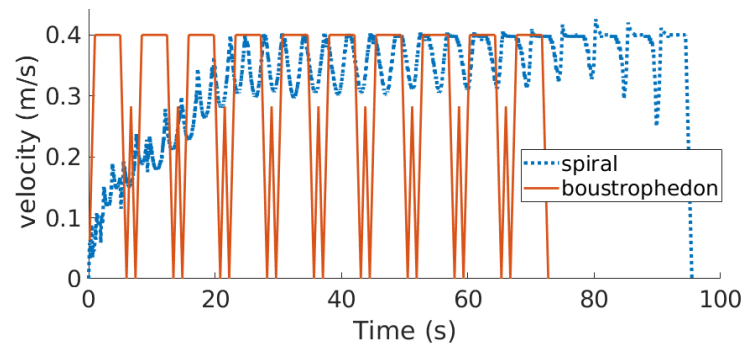

Fig. 7: Blasting spot velocity with respect to time. the lengths of the actual spiral path generated using DSCPP and the ideal spiral path is small.

Future work includes generalizing the DSCPP to a wider range of applications, testing it in real-world for various marine life, and deforming the spiral to various shapes using the mapping technique. Extending the algorithm to be applied to surfaces with complex curvatures will be investigated.

\section{ACKNOWLEDGEMENTS}

This work is supported in part by the Australian Research Council (ARC) Linkage Project (LP150100935), the Roads and Maritime Services of NSW, and the Centre for Autonomous Systems (CAS) at the University of Technology, Sydney.

\section{REFERENCES}

[1] E. Galceran and M. Carreras, "A survey on coverage path planning for robotics," Robotics and Autonomous Systems, vol. 61, no. 12, pp. 1258 - 1276, 2013

[2] G. Daoming and C. Jie, "ANFIS for high-pressure waterjet cleaning prediction," Surface and Coatings Technology, vol. 201, no. 3, pp. 1629 - 1634, 2006.

[3] A. W. Momber, "Chapter 3 - hydroblasting equipment," in Hydroblasting and Coating of Steel Structures, A. W. Momber, Ed. Oxford: Elsevier Science, 2003, pp. 45 - 75.

[4] C. Fong, "Analytical methods for squaring the disc," arXiv preprint arXiv:1509.06344, 2015

[5] F. Balampanis, I. Maza, and A. Ollero, "Spiral-like coverage path planning for multiple heterogeneous UAS operating in coastal regions," in International Conference on Unmanned Aircraft Systems, June 2017, pp. 617-624.

[6] T.-K. Lee, S.-H. Baek, Y.-H. Choi, and S.-Y. Oh, "Smooth coverage path planning and control of mobile robots based on high-resolution grid map representation," Robotics and Autonomous Systems, vol. 59, no. 10, pp. $801-812,2011$.

[7] Y. H. Choi, T. K. Lee, S. H. Baek, and S. Y. Oh, "Online complete coverage path planning for mobile robots based on linked spiral paths using constrained inverse distance transform," in International Conference on Intelligent Robots and Systems, 2009, pp. 5788-5793.

[8] T. K. Lee, S. H. Baek, S. Y. Oh, and Y. H. Choi, "Complete coverage algorithm based on linked smooth spiral paths for mobile robots," in International Conference on Control Automation Robotics Vision, Dec 2010, pp. 609-614.

[9] Y. Gabriely and E. Rimon, "Spiral-STC: an on-line coverage algorithm of grid environments by a mobile robot," in IEEE International Conference on Robotics and Automation, vol. 1, 2002, pp. 954-960.

[10] Y. Mei, Y.-H. Lu, Y. C. Hu, and C. S. G. Lee, "Energy-efficient motion planning for mobile robots," in IEEE International Conference on Robotics and Automation, vol. 5, April 2004, pp. 4344-4349.

[11] E. Gonzalez, P. Aristizabal, and M. Alarcon, "Backtracking spiral algorithm: a mobile robot region filling strategy," in International Symposium on Robotics and Automation, 2002, pp. 261-266.

[12] E. Gonzalez, O. Alvarez, Y. Diaz, C. Parra, and C. Bustacara, "BSA: A complete coverage algorithm," in IEEE International Conference on Robotics and Automation, April 2005, pp. 2040-2044.

[13] F. Hidalgo and T. Brunl, "Review of underwater SLAM techniques," in International Conference on Automation, Robotics and Applications, Feb 2015, pp. 306-311.

[14] D. Chaudhuri and A. Samal, "A simple method for fitting of bounding rectangle to closed regions," Pattern Recognition, vol. 40, no. 7, pp. 1981 - 1989, 2007

[15] M. Hassan, D. Liu, and G. Paul, "Collaboration of multiple autonomous industrial robots through optimal base placements," Journal of Intelligent \& Robotic Systems, vol. 90, no. 1-2, pp. 113-132, Oct 2017.

[16] M. Hassan, D. K. Liu, and G. Paul, "Modeling and stochastic optimization of complete coverage under uncertainties in multi-robot base placements," in International Conference on Intelligent Robots and Systems (IROS), Oct 2016, pp. 2978-2984. 